\title{
Amélioration des références et dissémination dans le domaine des hautes températures et de la pyrométrie optique au LNE-Cnam
}

\author{
Mohamed Sadli, Frédéric Bourson, Stephan Briaudeau, Jeanne-Marie Coutin, Thierry Deuzé, Guillaume Failleau, Séverine \\ Guimier, Stéphane Kosmalski, Olga Kozlova, Catherine Martin, Sid-Ali Mokdad, Lydia Rongione, Bernard Rougié, \\ Achour Sadouni, Daniel Truong, Vladimir Vidal et Yves Hermier
}

Laboratoire Commun de Métrologie, LNE-Cnam, 93210 Saint-Denis, France

\begin{abstract}
Résumé. Parmi les missions dévolues au Laboratoire Commun de Métrologie LNE-Cnam figurent la réalisation, l'amélioration et la dissémination de l'unité de température, le kelvin, sur tout le domaine de température. Dans le département « hautes températures et pyrométrie optique » cela concerne la thermométrie de contact au-dessus de $1000{ }^{\circ} \mathrm{C}$ et la pyrométrie optique sur tout le domaine de température. L'objectif de cet article est de permettre une vue d'ensemble des activités de recherche et d'étalonnage dans ce département ainsi que les projets transverses dans lesquels l'équipe est impliquée au-delà des limites des départements et des pôles du laboratoire. L'accent sera mis en particulier sur notre implication dans les projets européens de recherche concertée (JRP) qui nous engagent sur des durées de plusieurs années avec nos collègues européens sur des enjeux importante pour l'industrie et la société, tels que l'énergie, le système international d'unités et l'aide à l'industrie.
\end{abstract}

\section{Introduction}

Afin de préparer le mise-en-pratique de la nouvelle définition du kelvin dans le domaine des hautes températures et permettre d'intégrer des techniques nouvelles, le Comité Consultatif de Thermométrie (CCT) a officialisé la possibilité de disséminer la température thermodynamique directement ou via des points fixes à haute température [1]. Cela ne remet pas en cause l'utilisation de l'échelle internationale de température de 1990 (EIT-90) qui reste en vigueur et continuera d'être disséminée de manière routinière. Ces décisions sont le fruit des avancées notables réalisées dans deux domaines de recherche que sont la mesure de température par voie radiométrique et la réalisation de nouveaux points fixes à haute température. Le LNE-Cnam participe à ces avancées en consacrant, dans le cadre de la métrologie française, une partie de ses efforts à ces deux domaines depuis le début des années 2000.

Les actions de recherche s'inscrivent depuis plusieurs années dans un cadre européen et international avec les projets de recherche concertée (Joint Research Projects, JRP) notamment dans le cadre des projets InK (SIB01) et NOTED (SIB10) et des projets internationaux coordonnés par le groupe de travail $\mathrm{n}^{\circ} 5$ "pyrométrie optique" du CCT.

En parallèle à ces deux axes de recherche, des références de température et des capteurs sont développés

\footnotetext{
${ }^{\mathrm{a}}$ Corresponding author: mohamed.sadli@cnam.fr
}

pour la thermométrie par contact à haute température (audessus de $1000{ }^{\circ} \mathrm{C}$ ). Les principales actions de recherche concernent la mise en place de nouveaux points fixes adaptés aux thermocouples de type Platine/Palladium jusqu'à $1500{ }^{\circ} \mathrm{C}$ et aux nouveaux types de thermocouples tels que les thermocouples Platine-30\% Rhodium/Platine$60 \%$ Rhodium qui permettent de travailler jusqu'à $1800^{\circ} \mathrm{C}$ ou aux thermocouples de la famille Tungstène/Rhénium qui permettent d'atteindre jusqu'à $2300{ }^{\circ} \mathrm{C}$. L'adaptation de ces points fixes pour permettre l'étalonnage in-situ de thermocouples, via des méthodes d'auto-validation, est une des retombées de ces recherches qui nous permettent aujourd'hui de proposer des solutions innovantes dans ce domaine pour l'industrie, notamment dans le domaine du nucléaire.

La dissémination des références de température dans ce domaine est une des missions les plus importantes de notre laboratoire. Des prestations d'étalonnage en pyrométrie sont proposées entre $-20{ }^{\circ} \mathrm{C}$ et $1500{ }^{\circ} \mathrm{C}$ sous accréditation du COFRAC (Comité Français d'Accréditation) et nous préparons pour un avenir proche des prestations routinières jusqu'à des températures de $3000{ }^{\circ} \mathrm{C}$. Pour cela un four d'étalonnage a été étudié et caractérisé en termes d'émissivité et d'uniformité de température.

Cet article abordera chacune de ces quatre activités en général en renvoyant aux publications les plus récentes 
et les plus significatives de l'équipe afin d'approfondir le propos.

\section{Température thermodynamique}

Le LNE-Cnam met en œuvre depuis une dizaine d'année une méthode de mesure basée sur une mesure absolue de la luminance spectrale $[2,3]$. C'est une méthode qui permet de mesurer directement la température thermodynamique de points fixes de petites dimensions sans nécessité de corps noirs intermédiaires.

\subsection{Méthode de mesure de la température par voie radiométrique}

Une source monochromatique quasi lambertienne, accordable en longueur d'onde est utilisée pour étalonner la sensibilité spectrale du pyromètre «absolu». La luminance de cette source est déterminée à l'aide d'un luminancemètre raccordé au radiomètre cryogénique (référence nationale de flux lumineux) via un détecteur piège. En effet, le luminancemètre est constitué (voir figure1-d) de deux diaphragmes de dimensions connues (étalonnage nécessaire) séparés par une distance connue.

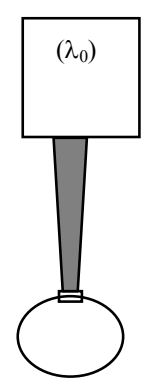

(a)

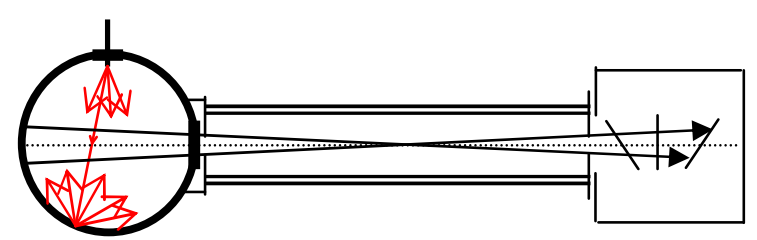

(d)

Figure 1. Principe de mesure de température thermodynamique d'un corps noir (a) Mesure de la luminance de sphère intégrante avec le luminancemètre (b) Mesure de la sensibilité spectrale du pyromètre avec la sphère intégrante (c) Mesure de la luminance du corps noir étudié avec le pyromètre (d) Détail du luminancemètre incluant la sphère intégrante et le détecteur étalon secondaire.

La source monochromatique quasi lambertienne est constituée d'un laser Ti:Sa continu accordable en longueur d'onde et injecté dans une sphère intégrante après couplage dans une fibre optique multimode. Cette fibre est agitée afin de réduire l'effet de speckle causé par la diffusion dans la sphère. La puissance du laser utilisé est stabilisée à mieux que $0,01 \%$ sur la durée de la comparaison du rayonnement du corps noir étudié à celui issu de la sphère intégrante.

Cette méthode a été mise en œuvre pour la mesure de la température thermodynamique des points eutectiques métal-carbone dans le cadre du projet de recherche concertée coordonné par le groupe de travail $\mathrm{n}^{\circ} 5$ «pyrométrie optique» du comité consultatif de thermométrie (CCT-WG5) [4].

L'affectation de la température thermodynamique a été effectuée via le point de cuivre puis par extrapolation aux points de $\mathrm{Co}-\mathrm{C}, \mathrm{Pt}-\mathrm{C}$ et $\mathrm{Re}-\mathrm{C}$. Les résultats que nous avons obtenus, présentés dans la table 1, étaient en accord avec les résultats des autres participants au projet [5].

Table 1. Températures thermodynamiques des points eutectiques metal-carbone $\mathrm{Co}-\mathrm{C}, \mathrm{Pt}-\mathrm{C}$ et $\mathrm{Re}-\mathrm{C}$ determinées par extrapolation à partir de la température thermodynamique du point fixe du cuivre au LNE-Cnam. Les cellules utilisées sont les cellules du LNE-Cnam : 2Co5, 5Pt1 et 4Re1.

\begin{tabular}{|c|c|c|}
\hline Point & $\begin{array}{c}\text { Températures } \\
\text { de fusion (K) }\end{array}$ & $\begin{array}{c}\text { Incertitude } \\
\text { type (mK) }\end{array}$ \\
\hline $\mathrm{Cu}$ & 1357.95 & 150 \\
\hline $\mathrm{Co}-\mathrm{C}$ & 1597.35 & 220 \\
\hline $\mathrm{Pt}-\mathrm{C}$ & 2011.52 & 280 \\
\hline $\mathrm{Re}-\mathrm{C}$ & 2747.74 & 680 \\
\hline
\end{tabular}

Ces premières mesures ont permis d'identifier les éléments à améliorer pour réduire les incertitudes et fiabiliser davantage la méthode de mesure. Nous avons en particulier à travailler sur la stabilité à moyen terme du comparateur de luminances, à diminuer les interréflexions et la diffusion dans le luminancemètre [6] et à caractériser plus précisément la distribution de luminance de la source monochromatique (sphère intégrante).

\subsection{Projets JRP InK SIB01 et NOTED SIB10}

Le LNE-Cnam contribue aux projets JRP InK « Implementing the new kelvin » coordonné par le NPL, et le projet NOTED « Novel Techniques for Traceable Temperature Dissemination ». Dans le cadre de ces deux projets, nous développons un pyromètre innovant qui permettra à terme d'envisager une alternative au comparateur de luminances et aux radiomètres à filtres pour réaliser un pyromètre «absolu» et qui pourra fonctionner en deux versions, visible et infrarouge, sur une gamme de température très large. Une thèse de doctorat est en cours au laboratoire sur ce sujet.

La mesure de la température thermodynamique de points fixes est un des objectifs du JRP InK tout comme la dissémination de l'unité de température via des points fixes à haute température.

Des cellules ont été réalisées, caractérisées et sélectionnées pour servir à ces mesures de température thermodynamique. Elles sont actuellement en circulation 
et nous devrions les recevoir et effectuer de nouvelles mesures en mai 2014 [7].

\section{Points fixes à haute température pour la pyrométrie optique}

La perspective de disposer d'une série de points fixes pyrométriques jusqu'à des températures de $2500{ }^{\circ} \mathrm{C}$ - et même au-delà - $[8]$ permet d'espérer un gain en incertitude d'un facteur 2 à 5 dans la réalisation du kelvin aux plus hautes températures. Il est d'ores et déjà possible d'utiliser les points fixes à haute température pour la mise-en-pratique de l'unité [1,9] en tant que références de température thermodynamique ou comme moyens de disséminer l'échelle de température.

Les travaux sur ces points fixes, basés sur les transitions eutectiques ou péritectiques métal-carbone, se poursuivent depuis les années 2000 dans le cadre de différents projets [10-17]. Cela a permis de réaliser et de caractériser plusieurs points fixes à haute température (Fe-C $\left(1153^{\circ} \mathrm{C}\right)$, Co-C $\left(1324{ }^{\circ} \mathrm{C}\right), \mathrm{Pd}-\mathrm{C}\left(1492{ }^{\circ} \mathrm{C}\right)$, Pt-C $\left(1738{ }^{\circ} \mathrm{C}\right)$, Re-C $\left(2474{ }^{\circ} \mathrm{C}\right)$. . etc. $)$ et surtout d'améliorer les méthodes de remplissage des cellules et leur design. La priorité que nous nous sommes fixée dès le début de ces travaux a été de réaliser les cellules les plus robustes possibles et d'optimiser la phase de remplissage pour minimiser les risques de pollution.

\subsection{Méthode de remplissage de cellules}

La méthode consiste à faire couler le mélange des matériaux préalablement préparé, depuis un entonnoir vers la cellule située au dessous. Le dispositif se présente comme une seringue (fig. 1-(a)) dont le piston pousse le matériau par gravité simple ou manuellement à l'aide d'une tige en graphite émergeant du four.

Après des évolutions multiples du dessin de nos cellules et de la méthode de remplissage, nous sommes parvenus au remplissage en une seule étape (pour les métaux disponibles en lingot ou granules) $[18,19]$. Ceci limite le temps d'exposition de l'alliage en fusion aux impuretés présentes dans le four.

Les cellules développées sont adaptées à une mise en œuvre dans des fours " haute température », en position horizontale pour une mesure pyrométrique. De forme cylindrique, elles n'excèdent pas $25 \mathrm{~mm}$ de diamètre et $40 \mathrm{~mm}$ de longueur. La cavité est d'une taille de $3 \mathrm{~mm}$ de diamètre et $30 \mathrm{~mm}$ de long. L'émissivité de cette cavité est estimée à $0,9997 \pm 2 \cdot 10^{-4}$.

Une des particularités de ces cellules est l'utilisation de feuilles composites $\mathrm{C} / \mathrm{C}$, qui permettent d'améliorer la conductivité thermique axiale autour du lingot. Elles sont associées à une " chemise " en graphite qui permet d'absorber, en se fissurant ou en cassant, les contraintes mécaniques dues aux dilatations différentes du graphite et de l'alliage (fig. 1-(b)). Cette conception s'est révélée particulièrement efficace pour améliorer la robustesse des cellules et la qualité des paliers de fusion des points eutectiques étudiés. En effet, des paliers de fusion significativement plus longs et présentant un domaine de fusion plus restreint ont été obtenus.

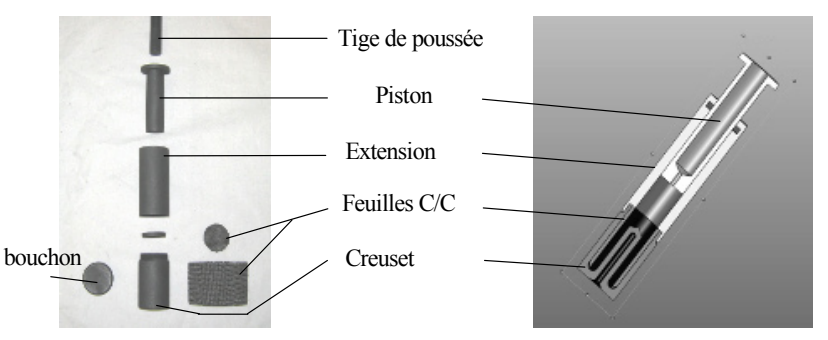

(a)

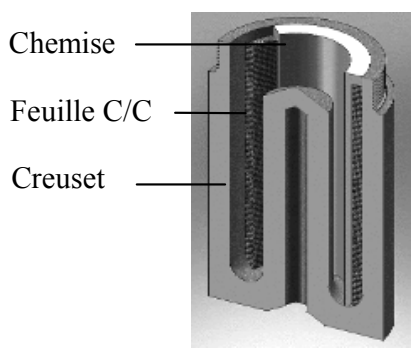

(b)

Figure 2. (a) Eléments en graphite constituant la cellule et l'ensemble de remplissage (b) Schéma en coupe de la cellule hybride (chemise + feuilles $\mathrm{C} / \mathrm{C}$ ).

\subsection{Cellules pour le projet InK}

Pour les besoins du projet InK, des cellules aux points de $\mathrm{Cu}\left(1084,62{ }^{\circ} \mathrm{C}\right)$, Co-C, Pt-C et Re-C ont été réalisées dans tous les laboratoires nationaux volontaires. Neuf laboratoires ont fourni des cellules: NPL, NMIJ, NRC, PTB, VNIIOFI, VNIIM, INRIM, PTB et LNE-Cnam. Nous avons fourni cinq cellules, soit au moins une cellule de chaque point, en respectant le cahier des charges strict préparé à cet effet. Ce cahier des charges détaille les critères d'acceptation de ces cellules en termes de pureté nominale des matériaux utilisés, des niveaux de température relatifs et des domaines de fusion [20].

Quatre laboratoires nationaux, un par point fixe, ont ensuite comparé et classé ces cellules sur des critères objectifs de qualité et de répétabilité de paliers et de niveau de températures de fusion obtenues. Le LNECnam a participé à ce processus de sélection en comparant toutes les cellules de Co-C.

Les résultats de la sélection à tous les points fixes ont été très satisfaisants pour le LNE-Cnam puisque toutes nos cellules ont été sélectionnées dans le lot des cinq cellules nécessaires par point fixe [21]. Ceci vient récompenser les efforts de développement et d'amélioration de nos cellules accomplis durant ces dernières années.

\subsection{Cellules pour le projet JRP HiTeMS IND01 et les applications industrielles}

Le LNE-Cnam participe au JRP HiTeMS IND01 « HighTemperature Measurement Solutions », coordonné par le NPL, sur la période 2011-2014 [22]. Parmi les actions qui nous incombent dans ce projet (voir $\$ 4.2$ pour ce qui concerne la thermométrie de contact) nous avons à 
réaliser des cellules pyrométriques adaptées à une utilisation industrielle en collaboration avec le laboratoire de maîtrise des accidents graves (LMA) au CEACadarache.

En partenariat avec le NPL et le Tubitak UME, nous avons développé des cellules points fixes à haute température pouvant fonctionner dans des conditions extrêmes d'utilisation.

Ces points fixes pourront être directement raccordés à l'EIT-90 de manière à proposer des références traçables destinées à être mises en œuvre in-situ. Le LNE-Cnam a proposé un nouveau dessin de creuset (figure 3) basé sur une structure hybride et une méthode de remplissage comparables à celles des cellules habituelles.

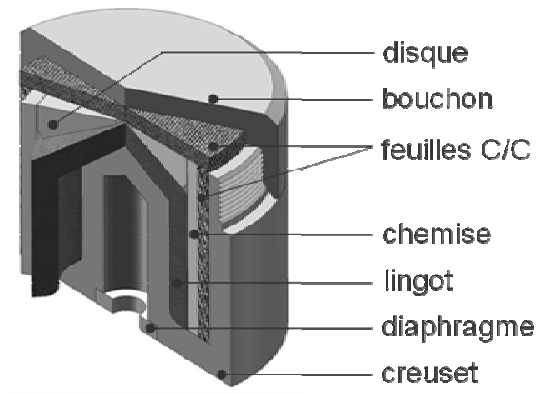

Figure 3. Vue en coupe des cellules HiTeMS pour application industrielle. La cellule est inspirée du design de nos cellules de référence -fig. $2-$ avec des parois plus épaisses pour augmenter la robustesse.

Le LNE-Cnam a fait fabriquer et a fourni des creusets aux deux laboratoires. Les dimensions extérieures réduites ainsi que l'élargissement de la cavité ont été expérimentés dans le but d'accroitre leur robustesse affectée par les variations de températures importantes du four à induction du LMA.

Des tests ont eu lieu sur ces cellules aux points de Co-C, Ru-C $\left(1953{ }^{\circ} \mathrm{C}\right)$ et Re-C d'abord dans les laboratoires nationaux dans des conditions thermiques optimales puis dans les installations du LMA dans un four à induction dans lequel des rampes de montée ou de descente en température de plusieurs milliers de degrés par minute ont été appliquées aux cellules sans dommage apparent. Les paliers de changement de phase sont même restés parfaitement exploitables dans ces conditions difficiles [23, 24].

Ainsi on peut voir que les utilisations des points fixes à haute température se trouvent autant dans le domaine le plus amont comme support de la température thermodynamique et comme moyen de dissémination de l'unité, que dans les domaines industriels où ils peuvent être transformés en moyens d'auto-validation et de réétalonnage in-situ.

\section{Thermocouples et méthodes d'auto- validation}

Les incertitudes d'étalonnage de thermocouples audessus du point de congélation du cuivre $\left(1084,64{ }^{\circ} \mathrm{C}\right)$ peuvent atteindre jusqu'à $\pm 1,5{ }^{\circ} \mathrm{C}$ à $1550{ }^{\circ} \mathrm{C}$ [25] en raison de l'absence de références de température fiables dans cette gamme de température.

La thermométrie de contact par thermocouples audessus de $1000{ }^{\circ} \mathrm{C}$ requiert donc des instruments et des références de température à même de garantir aux utilisateurs une meilleure exactitude et une traçabilité à l'échelle internationale de température. C'est dans cette optique que nous travaillons depuis une dizaine d'années au développement de thermocouples $\mathrm{Pt} / \mathrm{Pd}$ de très haute stabilité et de points fixes à haute température adaptés à leur étalonnage jusqu'à $1500{ }^{\circ} \mathrm{C}$ [26]. Au-delà de cette température, d'autres thermocouples peuvent être utilisés (W/Re, Ir/Ir-Rh, Pt-Rh/Pt-Rh..) mais leur stabilité aux plus hautes températures est insuffisante, rendant encore plus indispensable la disponibilité de références de température qui permettraient leur étalonnage et la correction de leur dérive en cours d'utilisation.

C'est pour répondre à ces objectifs que nous avons entrepris des travaux de recherche et de développement dans le cadre de projets européens et du programme de recherche de la métrologie française.

\subsection{Projet JRP MetroFission ENG08}

Le LNE-Cnam participe au JRP MetroFission (ENG08) financé dans le cadre de l'appel " Energie » du programme européen de recherche en métrologie l'EMRP (2009) sur la période 2010-2013 pour contribuer à trouver les réponses métrologiques aux besoins en termes de mesure des réacteurs nucléaires de nouvelle génération [28].

Une partie de ce projet est consacrée à développer des moyens et des méthodes pour la métrologie des températures qui soient compatibles avec un environnement nucléaire à des températures pouvant aller jusqu'à $1200{ }^{\circ} \mathrm{C}$ [29].

Dans ce cadre collaboratif, nous avons développé avec le NPL les premières cellules au point de $\mathrm{Fe}-\mathrm{C}$ pour la thermométrie de contact $[30,31]$ qui ont été caractérisées et comparées avec succès aux réalisations $\mathrm{du}$ NPL et même miniaturisées pour servir de moyens d'auto-validation [32]. En effet, la possibilité de disposer de ce type de références à points fixes dans les processus industriels permettant l'étalonnage régulier de capteurs de température est une demande récurrente de l'industrie. Il s'agit de proposer des solutions qui consistent à développer des modules points fixes pouvant être directement associés au capteur, avec un encombrement minimal, et compatibles avec la contrainte des atmosphères oxydantes, de manière à permettre un contrôle périodique in situ de la dérive de l'instrument.

Nous avons développé dans ce cadre et dans le cadre du projet HiTeMS des modules d'auto-validation divers pour couvrir la gamme de température allant de $960{ }^{\circ} \mathrm{C}$ à $1500{ }^{\circ} \mathrm{C}[33,34]$. La figure 4 montre un exemple de cellules d'auto-validation adaptées aux atmosphères inertes et qui a été testé aux points de l'argent $(961,78$ $\left.{ }^{\circ} \mathrm{C}\right)$, de l'or $\left(1064,18^{\circ} \mathrm{C}\right)$, du cuivre et du Fe-C. 


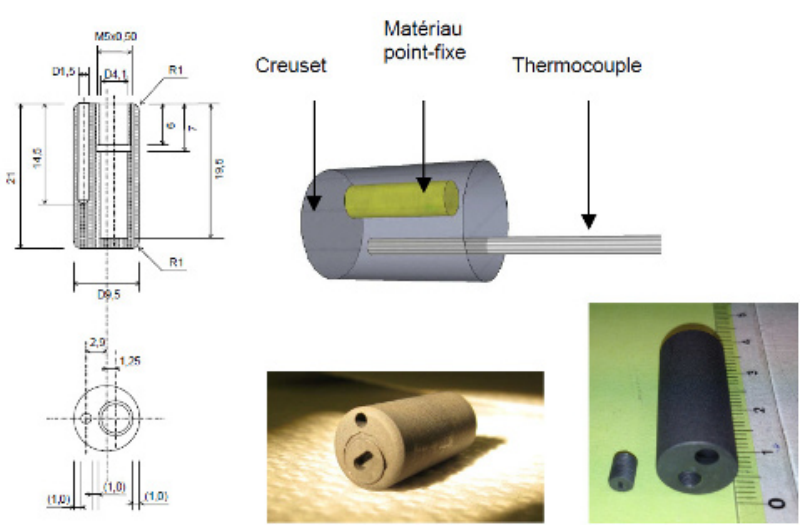

Figure 4. Cellule d'auto-validation en atmosphère inerte. Modèle avec lingot désaxé.

\subsection{Projet JRP HiTeMS IND01}

Dans le cadre de ce projet, nous contribuons au développement de moyens d'étalonnage jusqu'à $1800^{\circ} \mathrm{C}$, à la réalisation de moyens d'auto-validation en atmosphère oxydante pour thermocouples [34], à la caractérisation de thermocouples en termes de stabilité à haute température et de durée de vie et enfin, pour ce qui concerne la pyrométrie optique, au développement de cellules d'auto-validation pour pyromètres jusqu'à $2500{ }^{\circ} \mathrm{C}$ (voir plus haut $\$ 3.3$ ).

La figure 5 montre un exemple de technique d'autovalidation en atmosphère oxydante appliquée à un thermocouple de type B au point de l'or.
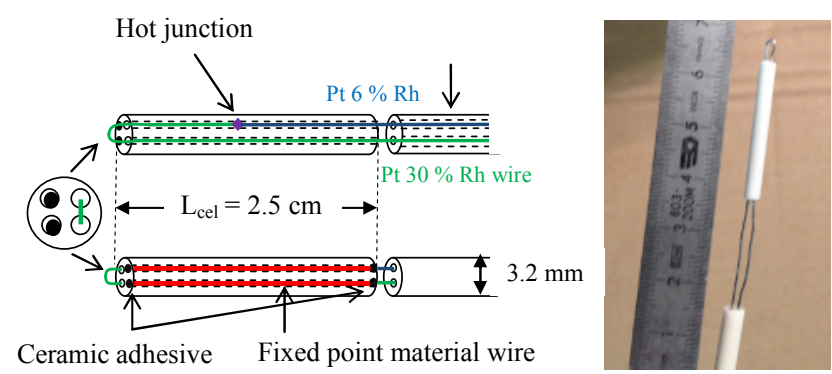

Figure 6. Dispositif d'auto-validation adapté aux atmosphères oxydantes pour thermocouple non chemisé.

Ainsi, des méthodes d'auto-validation originales sont progressivement mises en œuvre au laboratoire, et trouvent des applications concrètes dans le cadre du JRP HiTeMS et de la collaboration avec GDF-Suez et le CEA $[23,24]$

\section{Dissémination}

Au-dessus du point de congélation de l'argent (961,78 ${ }^{\circ} \mathrm{C}$ ), L'EIT-90 repose sur la loi du rayonnement de Planck. La température est déduite du rapport des luminances monochromatiques en prenant comme référence le rayonnement issu d'un corps noir au point fixe de l'argent, du cuivre $\left(1084,62{ }^{\circ} \mathrm{C}\right)$ ou de l'or $\left(1064,18{ }^{\circ} \mathrm{C}\right)$. Cela suppose donc une extrapolation depuis les températures de ces points fixes vers les températures les plus élevées.

La réalisation de l'échelle permet actuellement d'obtenir, dans les conditions courantes, des incertitudes de l'ordre de 1 à $2{ }^{\circ} \mathrm{C}$ autour de $2000{ }^{\circ} \mathrm{C}$ [35]. Cette incertitude est due principalement à l'extrapolation qui est effectuée entre le point de référence [36] et la température mesurée : l'instrument d'extrapolation, le pyromètre ou un comparateur de luminances, influe directement, et lourdement, sur cette incertitude via ses caractéristiques principales que sont sa sensibilité spectrale, sa linéarité et ses imperfections optiques (l'effet de taille de source qui provoque une sensibilité de la réponse à la taille de la source indépendamment de sa température).

Il est donc évident que de nouveaux points fixes de température répartis sur la gamme $1000{ }^{\circ} \mathrm{C}-3000{ }^{\circ} \mathrm{C}$ seraient une solution idéale pour réaliser l'échelle et mettre en pratique la nouvelle définition du kelvin [37].

La dissémination des références vers l'industrie et les utilisateurs est réalisée aujourd'hui par l'étalonnage de pyromètres ou de corps noirs entre $-20{ }^{\circ} \mathrm{C}$ et $1500{ }^{\circ} \mathrm{C}$ sous accréditation du comité français d'accréditation (COFRAC). Des nouvelles prestations entre $1500{ }^{\circ} \mathrm{C}$ et $3000{ }^{\circ} \mathrm{C}$ sont en préparation. Un four Thermogauge permettant de couvrir cette gamme de température a été caractérisé pour déterminer son émissivité et son uniformité de température. En effet, ces deux paramètres sont déterminants pour réduire les incertitudes de mesure. Différentes méthodes ont été mises en œuvre pour déterminer l'émissivité de la cavité rayonnante de ce four et les résultats obtenus par des mesures à plusieurs longueurs d'onde en se basant sur l'approximation de Wien de la loi de Planck sont présentés sur la figure 6 [38]. La valeur de l'émissivité de la cavité ainsi déterminée est estimée à $0,983 \pm 0,020$.

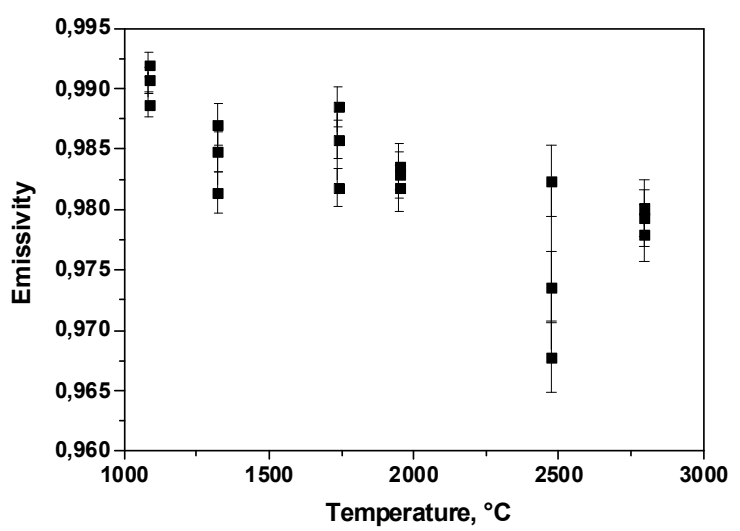

Figure 6. Valeurs de l'émissivité du four Thermogauge déduites de mesures à plusieurs longueurs d'onde.

Enfin, l'exploitation des points fixes à haute température pour la dissémination sera entreprise dans un futur proche puisqu'il est possible de mettre en œuvre ces cellules à points fixes avec des températures traçables à l'échelle comme moyens répétables de référence ou comme moyen de caractérisation de pyromètres. En effet, le fait de pouvoir vérifier l'exactitude de la réponse d'un pyromètre de référence en un ou deux points de manière 
routinière et pratique pourrait permettre d'élargir la périodicité d'étalonnage.

\section{Nouveaux développements}

Les projets de recherche européens sont fortement structurants et permettent d'inscrire nos actions de développement dans des thématiques liées à l'énergie, à l'industrie ou, plus en amont, à l'amélioration système international d'unités. C'est dans ce cadre qu'il s'agit aujourd'hui de projeter nos actions.

Un des besoins importants est l'amélioration des incertitudes d'étalonnage de pyromètres dans le domaine de température allant de $150{ }^{\circ} \mathrm{C}$ à $1000{ }^{\circ} \mathrm{C}$. Ce domaine intéresse beaucoup d'industries et souffre actuellement d'un chemin de traçabilité indirect. En effet, la traçabilité à l'échelle de température se fait, dans notre laboratoire, exclusivement via des thermomètres à résistance de platine ou des thermocouples. Nous projetons de développer des cavités à corps noirs points fixes dans ce domaine pour permettre une traçabilité directe à l'EIT-90 à travers les points fixes.

En plus de la réduction des incertitudes attendue après notre participation au projet Euramet 658 -extension [39], ces points fixes de référence serviront également comme supports à la température thermodynamique dans ce domaine de température.

Un banc de mesure incluant des fours à caloducs adaptés aux températures des points fixes d'indium, étain, zinc, aluminium et argent a été conçu à cet effet (fig. 7) et sera réalisé dans le futur proche si le financement est obtenu.

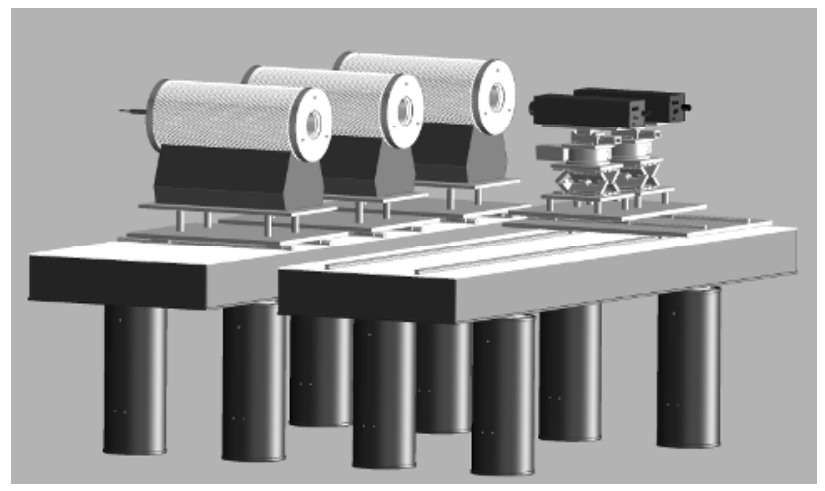

Figure 7. Vue schématique du banc envisagé avec une partie sources contenant les fours à caloducs pour les corps noirs points fixes, et une partie mesure avec des pyromètres pouvant être déplacés face aux sources.

\section{Conclusion}

Le paysage de la métrologie des hautes températures a été profondément bouleversé par l'arrivée des points eutectiques et péritectiques. Nous retrouverons dans l'avenir ces points fixes dans toutes les applications nécessitant des références de température jusqu'à $3000{ }^{\circ} \mathrm{C}$, que ce soit en amont pour «porter» et disséminer la température thermodynamique, ou dans les fours industriels quand il s'agira de surveiller et de corriger la dérive de capteurs de température.

Le LNE-Cnam s'est investi dans ce domaine et conforte une position de leader pour la réalisation de cellules pour la pyrométrie et pour la thermométrie de contact.

Le laboratoire fait également partie des rares laboratoires au monde à pouvoir mesurer la température thermodynamique par voie radiométrique. Il nous reste néanmoins à améliorer nos méthodes et moyens de mesure de la température thermodynamique à haute température et à les étendre, via une adaptation du radiomètre cryogénique aux grandes longueurs d'onde, aux températures moyennes. L'idéal étant de couvrir toute la gamme de température jusqu'à la jonction avec la thermométrie acoustique.

Le défi aujourd'hui est de mener à bien toutes ces actions dans le cadre de projets européens et nationaux. Ces actions même si elles peuvent paraître indépendantes, se nourrissent mutuellement et sont reliées ne serait-ce que par une traçabilité commune et des moyens communs.

La tendance de ces dernières années est à la baisse continue des crédits d'investissement et il semble hasardeux de parier sur une augmentation des moyens dans un avenir proche. Mais nous restons confiants dans l'utilité de nos développements pour l'industrie et pour la recherche.

\section{Remerciements}

Ces travaux ont été financés pour partie par le programme européen de recherche en métrologie (EMRP) pour ce qui concerne les JRP et, pour le restant, par la Métrologie Française sous l'égide du LNE.

\section{References}

1. G. Machin, K. Anhalt, P. Bloembergen, J. Hartmann, M. Sadli, P. Saunders, E. Woolliams, Y. Yamada, H. Yoon "Mise-en-Pratique of the Kelvin at High Temperature" CCT documents http://www.bipm.org/cc/CCT/Allowed/25/D12r_MePHT v8.pdf

http://www.bipm.org/cc/CCT/Allowed/25/D13_MePdirect method v3.1.pdf

http://www.bipm.org/cc/CCT/Allowed/25/D14_MePindirect method v3.1.pdf

2. S. Briaudeau, B. Rougie, M. Fanjeaux, M. Sadli, G. Bonnier "Thermodynamic temperature determination in high temperature range at BNMINM" Proceedings of 9th International Symposium on Temperature and Thermal Measurements in Industry and Science, Tempmeko '04, ed. D. Zvizdic, L.G. Bermanec, T. Stasic, T. Veliki, LMP/FSB, pp 119-125, Cavtat - Dubrovnik, 2004.

3. S. Briaudeau, M. Sadli, F. Bourson, B. Rougié, A. Rihan, J.-J. Zondy, "Primary Radiometry for the mise-enpratique: The Laser-Based Radiance Method Applied to a Pyrometer", Int. J. of Thermophysics 32, 2183- 2196 (2011). 
4. G. Machin, P. Bloembergen, J. Hartmann, M. Sadli, Y. Yamada "A concerted international project to establish high-temperature fixed points for primary thermometry", Int J Thermophysics, 28, 6, (2007), 1976-1982.

5. K. Anhalt, R.D. Taubert, G. Machin, E. Woolliams, M. R. Dury, H. Yoon, M. Sadli, S. Briaudeau, B. Khlevnoy, M. Ballico, F. Jahan, A. Todd, D. Woods “A comparison of absolute radiometry by metal-carbon eutectic fixed points" $9^{\text {th }}$ International Temperature Symposium, $17^{\text {th }}-23^{\text {rd }}$ March 2012, Anaheim, CA (USA).

6. S. Briaudeau, B. Rougié, M. Sadli, A. Richard et J.M. Coutin "Optical diffraction corrections in radiometric thermodynamic determination", $10^{\text {th }}$ International Symposium on Temperature and Thermal Measurements in Industry and Science (Tempmeko2007), Lake Louise, Canada, 21-25 mai 2007.

7. G. Machin, J. Engert, R. Gavioso, M. Sadli, E. Woolliams "The Euramet Metrology Research Programme Project Implementing the New Kelvin (InK)" soumis à Tempmeko 2013 (à paraître dans Int. J. Thermophysics).

8. Yamada Y, Sakate H, Sakuma F, Ono A, Proc. 7th Int. Symp. on Temperature and Thermal Measurements in Industry and Science (Tempmeko) pp 535-40, (1999).

9. G. Machin, P. Bloembergen, K. Anhalt, J. Hartmann, M. Sadli, P. Saunders, E. Woolliams, Y. Yamada, H. Yoon "Practical Implementation of the Mise en Pratique for the Definition of the Kelvin Above the Silver Point" Int. J. Thermophysics, 31, 1779-1788 (2010).

10. M. Sadli, G. Machin, D. Lowe, J. Hartmann, R. Morice, Proc. $8^{\text {th }}$ Int. Symp. On Temperature and Thermal Measurements in Industry and Science (Tempmeko) pp 507-12, (2001).

11. R. Morice, E. Devin, Proc. of Tempmeko '01, ed. B. Fellmuth, J. Seidel, G. Scholz, Berlin, VED VERLAG GMBH, 2002, pp 73 - 78 (2001).

12. G. Machin, G. Beynon, F. Edler, S. Fourrez, J. Hartmann, D. Lowe, R. Morice, M. Sadli, M. Villamañan, Proc. Temperature: Its Measurement and Control in Science and Industry, 7(Chicago, USA) AIP Conf. Proc. 684 285-90 (2002).

13. M. Sadli, M. Fanjeaux, G. Bonnier, Proc. Temperature: Its Measurement and Control in Science and Industry, 7 (Chicago, USA) AIP Conf. Proc. 684 267-72 (2002).

14. R. Morice, M. Megharfi, Favreau J.O., Morel E., Didialaoui I., Filtz J.R., Proc. of $9^{\text {th }}$ International Symposium on Temperature and Thermal Measurements in Industry and Science, Tempmeko '04, ed. D. Zvizdic et al, pp 847 - 852, Cavtat - Dubrovnik, 2004.

15. M. Sadli, F. Bourson, M. Fanjeaux, S. Briaudeau, B. Rougié, G. Bonnier "Study of metal-carbon eutectic points : from construction to temperature determination", Proc. of $9^{\text {th }}$ International Symposium on Temperature and Thermal Measurements in Industry and Science, Tempmeko '04, ed. D. Zvizdic et al, pp 611-616 (2004). 16. M. Sadli, F. Bourson, S. Briaudeau, T. Deuzé, G. Failleau, E. Agnandji, M. Ojanen, B. Rougié, "Review and prospects of the high temperature metrology activities at LNE-Cnam", proceedings électroniques du $15^{\text {ème }}$ Congrès International de Métrologie (Métrologie 2011).

17. M. Sadli, R. Morice, F. Bourson, S. Briaudeau, T. Deuzé, G. Failleau, J. Favreau, N. Fleurence, B. Rougié "Nouvelles Références à Haute Température à Base d'Alliages Eutectiques Métal-Carbone" Actes du Congrès de la Société Française de Thermique, Le Touquet 25-28 mai 2010.

18. F. Bourson, S. Briaudeau, B. Rougié, M. Sadli, Tempbeijing 2008, October 2008, Beijing, Popular Republic of China (2008).

19. M. Sadli, O. Pehlivan, F. Bourson, A. Diril, K. Ozcan, Int J Thermophys, 30, 36-46 (2009).

20. Y. Yamada, K. Anhalt, M. Battuello, P. Bloembergen, B. Khlevnoy, G. Machin, M. Matveyev, M. Sadli, T. Wang "Construction Of High-Temperature Fixed-Point Cells For Thermodynamic Temperature Assignment" $9^{\text {th }}$ International Temperature Symposium, $17^{\text {th }}-23^{\text {rd }}$ March 2012, Anaheim, CA (USA).

21. Y. Yamada, K. Anhalt, M. Battuello, P. Bloembergen, B. Khlevnoy, G. Machin, M. Matveyev, M. Sadli, A. Todd, T. Wang "Evaluation And Selection Of High-Temperature Fixed-Point Cells For Thermodynamic Temperature Assignment" soumis à Tempmeko 2013 (à paraître dans Int. J. Thermophysics).

22. G. Machin, K. Anhalt, F. Edler, J. Pearce, M. Sadli, R. Strnad, E. Vuelban "HITEMS: a pan-european project to solve high temperature measurement problems in industry", proceedings électroniques du $15^{\text {ème }}$ Congrès International de Métrologie (Métrologie 2011).

23. M. Sadli, T. Bellin-Croyat, F. Bourson, T. Deuzé, A. Diril, G. Failleau, C. Journeau, D. Lowe, S. Mokdad, M. Montero, C. Parga, N. Richard, "High-Temperature Fixed Points For Industrial Applications" soumis à Tempmeko 2013 (à paraître dans Int. J. Thermophysics). 24. M. Sadli, F. Bourson, A. Diril, D. Lowe, C. Journeau, C. Parga "Construction and in-situ characterisation of high-temperature fixed point cells devoted to industrial applications" dans ces actes.

25. L. Crovini, R. Perissi, J.W. Andrews, C. Brookes, W. Neubert, P. Bloembergen, G. Voyer, I. Wessel, High Temp. High Press. 19, 177 (1987).

26. R. Morice, F. Edler, J. Pearce, G. Machin, J. Fischer, J. R. Filtz "High-Temperature Fixed-Point Facilities for Improved Thermocouple CalibrationEuromet Project 857" Int J Thermophys, 29, 231-240 (2008).

27. P. Saunders, J. Fischer, M. Sadli, M. Battuello, C.W. Park, Y. Zundong, H. Yoon, W. Li, E. Van der Ham, F. Sakuma, J. Ishii, M. Ballico, G. Machin, N. Fox, J. Hollandt, M. Matveyev, P. Bloembergen, S. Ugur, "Uncertainty budgets for calibration of radiation thermometers below the silver point", Int. J. Thermophys., 29, 3, (2008), 1066-1083.

28. L. Johansson, J.-R. Filtz, P. DeFelice, M. Sadli, A. Plompen, B. Hay, A. Dinsdale, S. Pommé, P. Cassette, J. Keightley, "Advanced Metrology For New Generation Nuclear Power Plants", Proc. of 2nd IMEKO TCs $11,19,20$ on Metrological Infrastructure, Cavtat, Dubrovnik Riviera, Croatie, pp 77-80 (2011).

29. M. Sadli, M. de Podesta, D. del Campo, T. Deuzé, G. Edwards, C. J. Elliott, G. Failleau, S. Fourrez, C. 
Garcia Izquierdo, M. Laurie, S. A. Mokdad, J. V. Pearce, G. Sutton "New Temperature References and Sensors for the Next Generation of Nuclear Power Plants" International Conference ANIMMA 2013, 23-27 juin 2013, Marseille (2013).

30. Failleau G., Deuze T., Elliot C., Pearce J., Machin G., Briaudeau S., Sadli M., "Development of an Fe-C Eutectic Fixed-Point for the Calibration and In-Situ Monitoring of Thermocouples", Proc. of 2nd IMEKO TCs $11,19,20$ on Metrological Infrastructure, Cavtat, Dubrovnik Riviera, Croatie, pp 77-80 (2011).

31. C. J. Elliott, J. V. Pearce, G. Failleau, T. Deuzé, S. Briaudeau, M. Sadli, G. Machin, "Fe-C eutectic fixedpoint cells for contact thermometry: an investigation and comparison", Metrologia 49, 88-94 (2012).

32. C. J. Elliott, G. Failleau, T. Deuzé, M. Sadli, J. V. Pearce, G. Machin "Long-Term Monitoring of Thermocouple Stability with Miniature Fixed-Point Cells" soumis à Tempmeko 2013 (à paraître dans Int. J. Thermophysics).

33. G. Failleau, C.J. Elliott, T. Deuzé, J.V. Pearce, G. Machin, M. Sadli "Miniature Fixed-Point Cell Approaches For In-Situ Monitoring Of Thermocouple Stability" soumis à Tempmeko 2013 (à paraître dans Int. J. Thermophysics).

34. S. Mokdad, G. Failleau, T. Deuzé, S. Briaudeau, O. Kozlova, M. Sadli "A self-validation method for hightemperature thermocouples under oxidising atmospheres" soumis à Tempmeko 2013 (à paraître dans Int. J. Thermophysics).

35. J. Fischer et al. "Uncertainty budgets for realisation of scales by radiation thermometry" (2003) http://www.bipm.org/cc/CCT/Allowed/22/CCT03-03.pdf 36. Bourson F., Sadli M., Rougié B., Briaudeau S., "Study and Comparison of Newly Developed ITS-90 Silver- and Copper-Point Cells", Int. J. Thermophys., 32, 1602-1610 (2011).

37. P. Bloembergen, Y. Yamada, N. Yamamoto, J. Hartmann "Realizing the high-temperature part of a future ITS with the aid of eutectic metal-carbon fixed points" Temperature: Its Measurement and Control in Science and Industry vol. 7 ed D C Ripple (New York: AIP) pp 291-6 (2003).

38. O.Kozlova, S. Briaudeau, L.Rongione, F.Bourson, S.Guimier, S.Kosmalski, M.Sadli "Calibration of Radiation Thermometers up to $3000{ }^{\circ} \mathrm{C}$ : Effective Emissivity of the Source" soumis à Tempmeko 2013 (à paraître dans Int. J. Thermophysics).

39. H. C. McEvoy, M. J. Martin, A. Steiner, E. Schreiber, F. Girard, M. Battuello, M. Sadli, P. Ridoux, B. Gutschwager, J. Hollandt, A. Diril, Ö. Pehlivan "EURAMET Project to Examine Underlying Parameters in Radiance Temperature Scale Realisation, $156{ }^{\circ} \mathrm{C}$ to $1000{ }^{\circ} \mathrm{C}^{\prime}$ soumis à Tempmeko 2013 (à paraître dans Int. $J$. Thermophysics). 